\title{
Design of Networked Control Systems with Two Different Working Status
}

\author{
Guotao Hui ${ }^{1, *}$, Wanwei $\mathrm{Li}^{2}$ and Guangru Zhang ${ }^{3}$ \\ ${ }^{I}$ College of Information Science and Engineering, Northeastern University, Shenyang, Liaoning, 110819, P.R. China; \\ ${ }^{2}$ International Cooperation and Exchange Department, Northeastern University, Shenyang, Liaoning, 110819, P.R. \\ China; ${ }^{3}$ State Grid Gansu Electric Power Research Institute, Lanzhou, Gansu, 730050, P.R. China
}

\begin{abstract}
This paper is concerned with stability and controller design of networked control systems (NCSs) with two different working status. Firstly, the working status of NCSs is considered as two different cases: the normal case and the unnormal case. In normal case, the work process of NCSs is described as two independent working processes: one is the packet dropouts process; the other is the network induced delay process. In un-normal case, there are two goals that should be achieved for designing: one is to simulate the initial system for restarting the normal case; the other is to ensure the stability of NCSs as much as possible. Secondly, according to the designed networked controller, a novel necessary and sufficient condition for guaranteeing the stability of the NCSs under the un-normal case has been proposed. Furthermore, the dynamic behavior of the NCSs which has switching between the normal case and the un-normal case, has been considered. Finally, a numerical example illustrates the effectiveness of the results.
\end{abstract}

Keywords: Necessary and Sufficient Conditions, Network-Induced Delay, Nonlinear Switched Systems, Packet Dropouts, Restarting, Controller.

\section{INTRODUCTION}

A typical NCSs can be considered as a sampled-data feedback control system with communication network [1]. It is well known that the use of NCSs has many considerable advantages in practice, such as reduced system wiring, low weight, easy on system diagnosis and maintenance [2]. But the communication network itself is a dynamical system including some issues, such as data packet dropouts, limited bandwidth, time-delay, and quantization, which might be potential sources of instability and poor performance of control systems [3]. Therefore, during the last decade, a great deal of studies have been developed for the analysis and synthesis of NCSs, for example, see [4-6].

Although NCSs have been studied by many researchers for a number of years, there are still some interesting problems that deserved further research. In particular, the socalled multi-rate technique has been successfully developed and widely used in modern industry. In [7], the authors firstly concerned about the design problem of NCS with piecewise constant generalized sampled-data hold (PCGSHF). They pointed out that there are many remarkable advantages for employing PCGSHF in lieu of ZOH. The main reason of these advantages is that the hold function itself is the design variable instead of a given constant (such as $\mathrm{ZOH}$ ). Correspondingly, the additional design degree-of-freedom can be gained for improving the system performance. The study in this paper can be considered as the development of the theory in [7]. It should be mentioned that due to the added design degree-of-freedom by introducing the PCGSHF, the design

*Address correspondence to this author at the College of Information Science and Engineering, Northeastern University, Shenyang, Liaoning, 110819, P.R. China; Tel: 13898863791; E-mail: huiguotao@vip.163.com strategy which is based on the characteristics of the communication, network may be improved. It is well known that because of the complexity of network environment, some given characteristics of the communication network may change occasionally. For example, according to the actual testing situation, a series of parameters for describing the characteristics of the communication network can be confirmed. Correspondingly, all of the system components are designed based on these conditions. However, sometimes in actual operation, the considered conditions may be incomplete, which leads to the failure mode. In fact, a better control strategy which is based on conditions, is not only related to the given conditions, but is also perfect for fault tolerant, which is induced by unexpected conditions. Here it should be noted that the existing results for studying fault-tolerant control problem of NCSs have concentrated on the fault of system components rather than the given characters of network, for instance, see $[8,9]$. So it is necessary to propose an effective strategy for handling the fault, which is caused by the variation of given design condition. Such observation motivates the current study.

In this paper, we consider the stability and controller design problem of networked control systems (NCSs) with two different working status. The obtained results can be considered as the development of the theory in [7], which only considered the normal case. Under consideration of networkinduced delay and data packet dropout phenomenon in the transmission, the system model which includes two work patterns (the normal and the un-normal) for the NCSs with PCGSHF has been proposed. Under the normal case, both the networked controller and the weights of PCGSHF for ensuring stability of NCSs are designed according to [7]. The un-normal case is based on the unexpected conditions, which are in contrast with the given characters of communication 
network. There are two goals that should be achieved for designing: one is to simulate the initial system for restarting the normal case; the other is to ensure the stability of NCSs as much as possible. So a novel, necessary and sufficient condition for guaranteeing the stability of the NCSs under the un-normal case has been proposed. Furthermore, the dynamic behavior of the NCSs which is switching between the normal case and the un-normal case is considered. Finally, a numerical example illustrates the effectiveness of the results.

\section{PRELIMINARIES}

In this paper, the plant of NCSs is considered as

$\dot{x}(t)=A x(t)+B u(t)$

where $x(t) \in \mathfrak{R}^{n}$, and $u(t) \in \mathfrak{R}^{m} . A$ and $B$ are known constant matrices and ensure the plant (1) is controllable. The controller is event-driven and of the form

$u(k h)=F x(k h)$

where $F \in \mathfrak{R}^{m \times n}$, and $k \in Z$. With regard to the network environment, $\tau_{s c}$ and $\tau_{c a}$ are respectively used to describe the sensor-to-controller delay and the controller-to-actuator delay. The actuator is event-driven and the sensor is timedriven with sampling period $h$. Moreover, the following assumptions are needed for the considered NCSs:

A1. If the transmissive delay $\tau=\tau_{s c}+\tau_{c a}$ satisfies $0<\tau \leq \tau_{\max }<h$, the input signals $u(k h-\tau)$ are considered as successful transmission.

A2. If the transmissive delay $\tau=\tau_{s c}+\tau_{c a}$ does not satisfy $0<\tau \leq \tau_{\max }<h$, the input signals $u(k h-\tau)$ are considered as unsuccessful transmission, which means packet dropout. Here the number of successive packet dropout is upper bounded, and the bound is denoted by $\mathrm{d}_{\max }$ which is a known constant.

These assumptions were taken into account in the $[7,10$ 12]. According to the above assumptions, it is obvious that the operative mode of NCSs is constituted by two cases, the successful transmissions and the un-successful transmissions. With regard to the successful transmissions, a set $\Pi=\left\{\alpha_{i} \mid \alpha_{i} \in k h ; i, k \in Z\right\}$ is considered and denoted the sequence points of successful transmissions from the sensor to the actuator. Correspondingly, according to [7], the model of NCS in normal case can be obtained,

$$
\begin{aligned}
& x\left(\alpha_{i+1}\right)=G\left(d\left(\alpha_{i}\right), w_{i}\right) x\left(\alpha_{i}\right) \\
& +H\left(d\left(\alpha_{i}\right), w_{i}\right) x\left(\alpha_{i-1}\right) \\
& x\left(\alpha_{1}\right)=G\left(d\left(\alpha_{0}\right), w_{0}\right) x\left(\alpha_{0}\right)
\end{aligned}
$$

in which

$$
\begin{aligned}
& G\left(d\left(\alpha_{i}\right), w_{i}\right) \\
& =e^{A d\left(\alpha_{i}\right) h}\left(e^{A h}+M_{1}\left(w_{i}\right)+M\left(w_{i}\right)\right) \\
& +\vartheta\left(d\left(\alpha_{i}\right)\right) \times \\
& \sum_{\beta=0}^{d\left(\alpha_{\alpha}\right)-1}\left(e^{A h}\right)^{\beta}\left(M_{1}\left(w_{i}\right)+\hat{N}\left(w_{i}\right)\right) \\
& \tau\left(\alpha_{i}\right) \in\left(\left(w_{i}-1\right) \frac{h}{\bar{a}}, w_{i} \frac{h}{\bar{a}}\right], \\
& M_{1}\left(w_{i}\right)=\sum_{c=w+1}^{\bar{a}} \int_{(c-1) \frac{h}{\bar{a}}}^{c \frac{h}{\bar{a}}} e^{A(h-s)} d s B \delta(c) F, \\
& H\left(d\left(\alpha_{i}\right), w_{i}\right)=e^{A d\left(\alpha_{i}\right) h} \hat{N}\left(w_{i}\right)-e^{A d\left(\alpha_{i}\right)_{h}} M\left(w_{i}\right), \\
& \Omega=\left\{0,1,2, \cdots, d_{\max }\right\}, \\
& \hat{N}\left(w_{i}\right)=\sum_{c=1}^{w_{i}} \int_{(c-1) \frac{h}{\bar{a}}}^{c \frac{h}{\bar{a}}} e^{A(h-s)} d s B \delta(c) F, \\
& M\left(w_{i}\right)=\int_{\tau\left(\alpha_{i}\right)}^{w \cdot \frac{h}{\bar{a}}} e^{A(h-s)} d s B \delta\left(w_{i}\right) F, \\
& \vartheta\left(d\left(\alpha_{i}\right)\right)=\left\{\begin{array}{l}
d\left(\alpha_{i}\right)=0, \\
d\left(\alpha_{i}\right) \neq 0 .
\end{array} d\left(\alpha_{i}\right)=\frac{\left(\alpha_{i+1}-\alpha_{i}\right)}{h}-1,\right. \\
& d\left(\alpha_{i}\right) \in \Omega, w_{i} \in\left\{, 2, \cdots \frac{\tau_{\max } \bar{a}}{h}\right\} \bar{a}=\frac{h}{\hat{a} \times 10^{-a}}, \\
& h(t)=\delta(s), k h+\frac{(s-1) h}{\bar{a}} \leq t<k h+\frac{s h}{\bar{a}},
\end{aligned}
$$

$s \in\{1,2, \cdots \bar{a}\}, \bar{a}$ is the number of segments for PCGSHF during one sampling period.

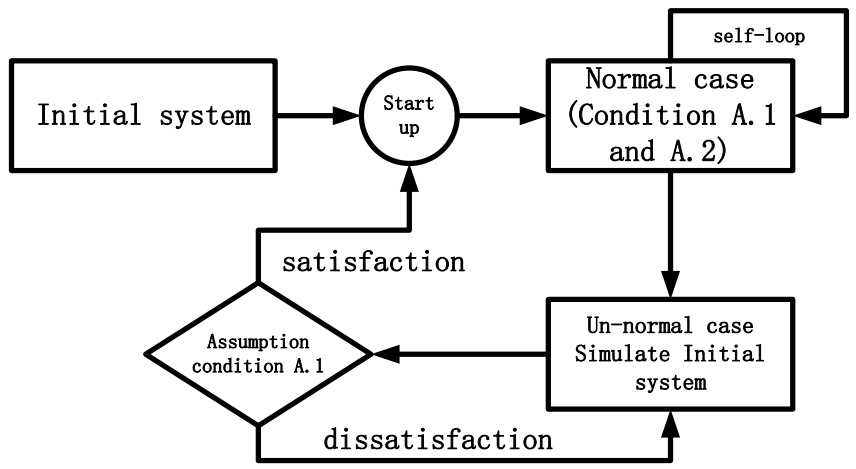

Fig. (1). The working status of NCSs.

Here it should be noted that the obtained (3) and (4) are based on the assumptions A1 and A2 (the normal case in Fig. 1). Although the above assumptions can accord with network environment as far as possible, sometimes the complex net- 
work environment in practical application may generate some extreme cases. For instance, according to the A2, the new control signal $u\left(\alpha_{i}+\left(d_{\max }+1\right) h\right)$ must arrive at the actuator during the interval $\left(\alpha_{+}+\left(d_{\max }+1\right) h, \alpha_{+}+\right.$ $\left.\left(d_{\max }+1\right) h+\tau_{\max }\right]$ because the maximum number of packet dropouts is $d_{\text {max }}$. However, who can ensure that the signal must arrive at the actuator during the considered interval? So if the extreme case occurs, we consider the working status of NCSs enter into the un-normal case, which is described as in Fig. (1). In un-normal case, there are two goals that should be achieved: one is to simulate the initial system for restarting the normal case; the other is to ensure the stability of NCSs as much as possible. Based on both the goals, the control strategy can be designed as follows: At first, we assume the first time the un-normal case appears at the instant $\alpha_{i}+\left(d_{\text {max }}+1\right) h$, Correspondingly, the control strategy can be designed as

$$
\begin{aligned}
& x\left(\alpha_{i}+\left(d_{\max }+1\right) h+h\right) \\
& =e^{A h} x\left(\alpha_{i}+\left(d_{\max }+1\right) h\right) \\
& +\sum_{c=1}^{\frac{\tau_{m}}{a}} \int_{(c-1) \frac{h}{\bar{a}}}^{c} e^{A-\bar{a}} \\
& +L(0) x\left(\alpha_{i}\right)
\end{aligned}
$$

where $L(j)=\sum_{\substack{c=\bar{a} \\ c=\frac{c_{-}}{h}+1}}^{\bar{a}} m_{j}(c) \int_{(c-1) \frac{h}{\bar{a}}}^{c} e^{\frac{h}{\bar{a}}} e^{A(h-s)} d s B \delta(c) F$,

$j \in Z$ and $m_{j}(c) \in\{1,0\}$. Furthermore, in the next sampling interval, we have

$$
\begin{aligned}
& x\left(\alpha_{i}+\left(d_{\text {max }}+1\right) h+2 h\right)= \\
& e^{A h} x\left(\alpha_{i}+\left(d_{\text {max }}+1\right) h+h\right) \\
& +L(1) x\left(\alpha_{i}\right)
\end{aligned}
$$

According to (5) and (6), it is obvious that the control signal $u\left(\alpha_{i}\right)$ be placed by the signal $0 \in \mathfrak{R}^{m}$ at $\alpha_{i}+\left(d_{\text {max }}+1\right) h+h$. Such consideration mainly realizes the control inputs of initial system (4) for simulating the initial control status of the normal case. If the control signal $u\left(\alpha_{i}+\left(d_{\max }+1\right) h+h\right)$ arrives at the actuator during $\left(\alpha_{i}+\left(d_{\text {max }}+1\right) h+h, \alpha_{i}+\left(d_{\text {max }}+1\right) h+h+\tau_{\text {max }}\right]$, the NCSs can be considered to restart in normal case (the initial state is $x\left(\alpha_{i}+\left(d_{\max }+1\right) h+h\right)$ and the initial input is $\left.0 \in \mathfrak{R}^{m}\right)$. Moreover, the control signal $u\left(\alpha_{t}\right)$ is used once more during the intervals $\left(\alpha_{i}+\left(d_{\text {max }}+1\right) h+\tau_{\text {max }}\right.$, $\left.\alpha_{i}+\left(d_{\text {max }}+1\right) h+h\right)$ and $\left(\alpha_{i}+\left(d_{\text {max }}+2\right) h+\tau_{\text {max }}\right.$, $\left.\alpha_{i}+\left(d_{\text {max }}+2\right) h+h\right)$.

There are two reasons for doing that: one is we only have $u\left(\alpha_{i}\right)$ can be used, even if it is an old signal; the other is that if we don't use $u\left(\alpha_{i}\right)$, the system model of NCSs in no-normal case is an autonomous system. Correspondingly, the system stability cannot be guaranteed when $\left\|e^{A h}\right\| \geq 1$. Therefore, in the following analysis, with regard to the normal case, the PCGSHF $h(\mathrm{t})$ and the networked state feedback controller $F$ can be designed for guaranteeing the stability of the NCSs (3) by using the criterion in [7]. With regard to the un-normal case, the main object of this paper is proposed as an effective algorithm which satisfies necessary and sufficient condition for obtaining the maximum allowable refresh time of the designed NCSs [13-15].

\section{MAIN RESULTS}

Theorem 1. Let $\bar{\alpha}_{0} \in \Pi$ describe the initial instant $\alpha_{0}$ or the initial instant from the un-normal case to the normal case. Correspondingly, $u\left(\bar{\alpha}_{i}\right)=F x\left(\bar{\alpha}_{i}\right)$ is used to describe the latest control input from the initial instant $\bar{\alpha}_{0}$ to the un-normal case. The necessary and sufficient condition for guaranteeing the stability of the NCSs under the unnormal case is that there exist $L(j)(j \in Z)$ which satisfies

$\left|\lambda_{\text {max }}(\bar{Z}(\zeta))\right|<1$

and

$$
\begin{aligned}
& \bar{Z}(\zeta)=e^{A \zeta h h} G\left(d_{\text {max }}, \bar{w}_{i}\right) Z\left(\bar{\alpha}_{i-1}, \cdots \bar{\alpha}_{0}\right) \\
& +e^{A \zeta h h} G\left(d_{\max }, \bar{w}_{i}\right) Z\left(\bar{\alpha}_{i-2}, \cdots \bar{\alpha}_{0}\right) \\
& +e^{A(\zeta-1)_{h}} \times \\
& \sum_{c=1}^{\frac{\tau_{m} \bar{a}}{h}} \int_{(c-1) \frac{h}{\bar{a}}}^{c \frac{h}{a}} e^{A(h-s)} d s B \delta(c) F Z\left(\bar{\alpha}_{i-1}, \cdots \bar{\alpha}_{0}\right) \\
& +\sum_{j=0}^{\zeta-1} e^{A(\zeta-j-1) h} L(j) Z\left(\bar{\alpha}_{i-1}, \cdots \bar{\alpha}_{0}\right)
\end{aligned}
$$




$$
\begin{aligned}
& Z\left(\bar{\alpha}_{i-2}, \cdots \bar{\alpha}_{0}\right)= \\
& {\left[G\left(d\left(\bar{\alpha}_{i-2}\right), \bar{w}_{i-2}\right) \quad H\left(d\left(\bar{\alpha}_{i-2}\right), \bar{w}_{i-2}\right)\right]} \\
& \times \\
& \prod_{y=i-3}^{1}\left[\begin{array}{cc}
G\left(d\left(\bar{\alpha}_{y}\right), \bar{w}_{y}\right) & H\left(d\left(\bar{\alpha}_{y}\right), \bar{w}_{y}\right) \\
I & 0
\end{array}\right] \\
& \times\left[\begin{array}{c}
G\left(d\left(\bar{\alpha}_{0}\right), \bar{w}_{0}\right) \\
I
\end{array}\right] \\
& \text { and } \\
& Z\left(\bar{\alpha}_{i-1}, \cdots \bar{\alpha}_{0}\right)= \\
& {\left[G\left(d\left(\bar{\alpha}_{i-1}\right), \bar{w}_{i-1}\right) \quad H\left(d\left(\bar{\alpha}_{i-1}\right), \bar{w}_{i-1}\right)\right]} \\
& \times \\
& \prod_{y=i-2}^{1}\left[\begin{array}{cc}
G\left(d\left(\bar{\alpha}_{y}\right), \bar{w}_{y}\right) & H\left(d\left(\bar{\alpha}_{y}\right), \bar{w}_{y}\right) \\
I & 0
\end{array}\right] \\
& \times\left[\begin{array}{c}
G\left(d\left(\bar{\alpha}_{0}\right), \bar{w}_{0}\right) \\
I
\end{array}\right]
\end{aligned}
$$

Proof: From (3) and (4), we have

$$
\begin{aligned}
& x\left(\bar{\alpha}_{i}+\left(d_{\text {max }}+1\right) h\right)=G\left(d_{\text {max }}, \bar{w}_{i}\right) x\left(\bar{\alpha}_{i}\right) \\
& +H\left(d_{\max }, \bar{w}_{i}\right) x\left(\bar{\alpha}_{i-1}\right)
\end{aligned}
$$

and

$$
\begin{aligned}
& x\left(\bar{\alpha}_{1}\right)=G\left(d\left(\bar{\alpha}_{0}\right), \bar{w}_{0}\right) x\left(\bar{\alpha}_{0}\right) \\
& x\left(\bar{\alpha}_{2}\right)=G\left(d\left(\bar{\alpha}_{1}\right), \bar{w}_{1}\right) x\left(\bar{\alpha}_{1}\right)+ \\
& H\left(d\left(\bar{\alpha}_{1}\right), \bar{w}_{1}\right) x\left(\bar{\alpha}_{0}\right)
\end{aligned}
$$$$
x\left(\bar{\alpha}_{i}\right)=G\left(d\left(\bar{\alpha}_{i-1}\right), \bar{w}_{i-1}\right) x\left(\bar{\alpha}_{i-1}\right)
$$$$
+H\left(d\left(\bar{\alpha}_{i-1}\right), \bar{w}_{i-1}\right) x\left(\bar{\alpha}_{i-2}\right)
$$

By using the iteration of (10), there exists

By using the iteration of (11),

Furthermore, according to the proposed control strategies (9) and (10), we have

By using the iteration of (13) and (14), we can obtain $x\left(\overline{\alpha_{i}}\right)=$

$\left[G\left(d\left(\bar{\alpha}_{i-1}\right), \bar{w}_{i-1}\right) \quad H\left(d\left(\overline{\alpha_{i-1}}\right), \bar{w}_{i-1}\right)\right]_{\times}$

$\left[\begin{array}{l}x\left(\bar{\alpha}_{i-1}\right) \\ x\left(\bar{\alpha}_{i-2}\right)\end{array}\right]=$

$\left[G\left(d\left(\bar{\alpha}_{i-1}\right), \bar{w}_{i-1}\right) \quad H\left(d\left(\bar{\alpha}_{i-1}\right), \bar{w}_{i-1}\right)\right] \times$

$\left[\begin{array}{cc}G\left(d\left(\bar{\alpha}_{i-2}\right), \bar{w}_{i-2}\right) & H\left(d\left(\overline{\alpha_{i-2}}\right), \bar{w}_{i-2}\right) \\ I & 0\end{array}\right] \times$

$\left[\begin{array}{l}x\left(\bar{\alpha}_{i-2}\right) \\ x\left(\bar{\alpha}_{i-3}\right)\end{array}\right]$

$x\left(\bar{\alpha}_{i}+\left(d_{\text {max }}+1\right) h\right)=$

$G\left(d_{\max }, \bar{w}_{i}\right) Z\left(\bar{\alpha}_{i-1}, \cdots \bar{\alpha}_{0}\right) x\left(\bar{\alpha}_{0}\right)$

$+H\left(d_{\max }, \bar{w}_{i}\right) Z\left(\bar{\alpha}_{i-2}, \cdots \bar{\alpha}_{0}\right) x\left(\bar{\alpha}_{0}\right)$

$x\left(\bar{\alpha}_{i}+\left(d_{\max }+1\right) h+h\right)=$

$e^{A h} G\left(d_{\text {max }}, \bar{w}_{i}\right) Z\left(\bar{\alpha}_{i-1}, \cdots \bar{\alpha}_{0}\right) x\left(\bar{\alpha}_{0}\right)$

$+e^{4 h} H\left(d_{\max }, \bar{w}_{i}\right) Z\left(\bar{\alpha}_{i-2}, \cdots \bar{\alpha}_{0}\right) x\left(\bar{\alpha}_{0}\right)+$

$\sum_{c=1}^{\frac{\tau_{m} \bar{a}}{h}} \int_{(c-1) \frac{h}{\bar{a}}}^{c_{\bar{a}}^{h}} e^{\lambda(h-s)} d s B \delta(c) F Z\left(\bar{\alpha}_{i-1}, \cdots \overline{\alpha_{0}}\right) x\left(\bar{\alpha}_{0}\right)$

$+L(0) Z\left(\bar{\alpha}_{i-1}, \cdots \bar{\alpha}_{0}\right) x\left(\bar{\alpha}_{0}\right)$

and

$x\left(\bar{\alpha}_{i}+\left(d_{\text {max }}+1\right) h+2 h\right)=$

$e^{A h} x\left(\bar{\alpha}_{i}+\left(d_{\max }+1\right) h+h\right) x\left(\bar{\alpha}_{0}\right)$

$+L(1) Z\left(\bar{\alpha}_{i-1}, \cdots \bar{\alpha}_{0}\right) x\left(\bar{\alpha}_{0}\right)$

$x\left(\bar{\alpha}_{i}+\left(d_{\text {max }}+1\right) h+\zeta h\right)=\bar{Z}(\zeta) x\left(\bar{\alpha}_{0}\right)$

in which $\zeta \in Z^{+}, \bar{Z}(\zeta)$ is shown in (7).

Therefore, based on the (7), it is obvious that the necessary and sufficient condition for guaranteeing the stability of the NCSs under the un-normal case is that there exists $L(j)(j \in Z)$ which satisfies $\left|\lambda_{\text {max }}(\bar{Z}(\zeta))\right|<1$.

\section{EXAMPLE}

In this section, we present a numerical simulation to show the application of the proposed method in this paper. 


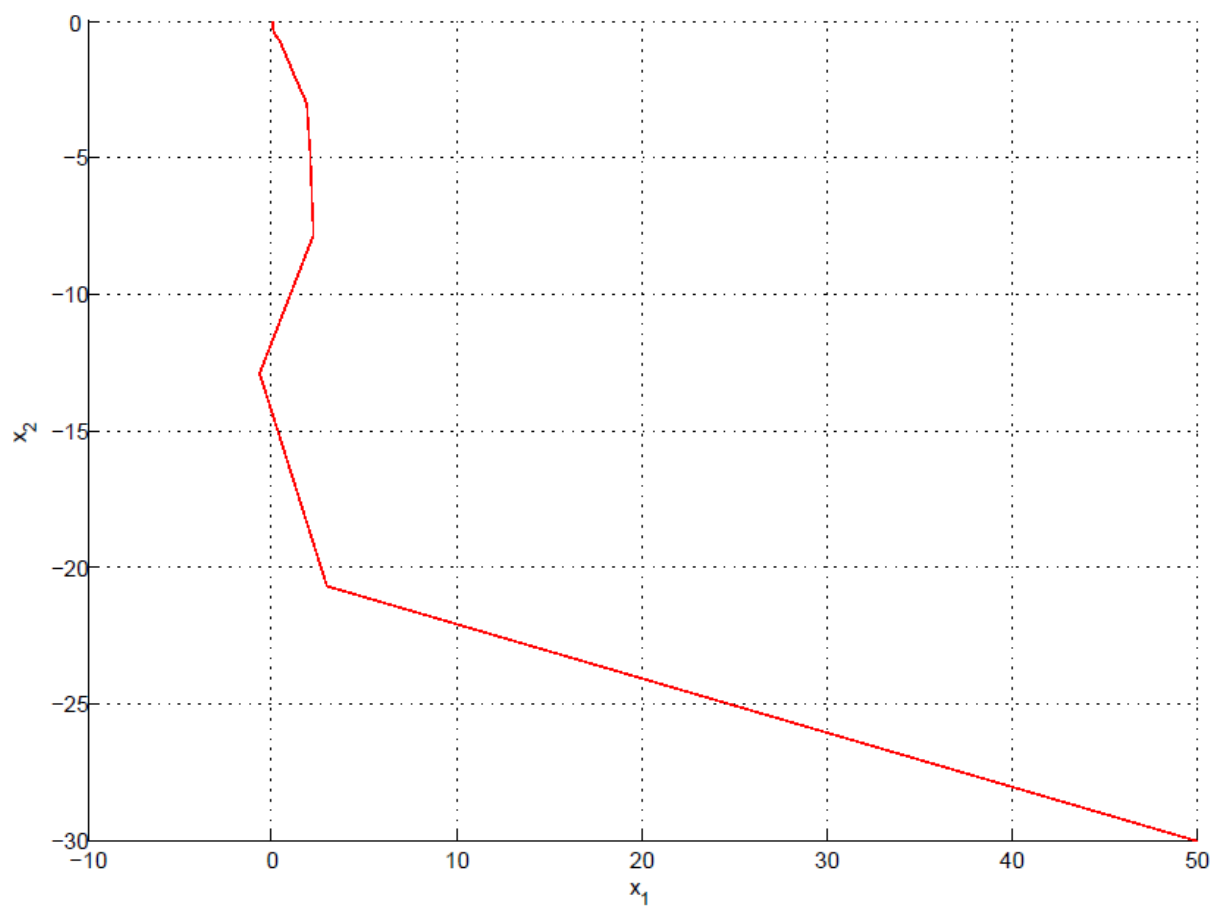

Fig. (2). State response curve of NCSs with initial conditions.

Considering the continuous-time linear time-invariant model as

$\dot{x}(t)=\left[\begin{array}{cc}0 & 1 \\ 0 & -0.1\end{array}\right] x(t)+\left[\begin{array}{c}0 \\ 0.1\end{array}\right] u(t)$

At first, some basic conditions are given as follows: $h=1 \mathrm{~s}, \quad d_{\max }=4, \quad \tau_{\max }=0.7 \mathrm{~s}, \quad \kappa=3, \quad$ and $\left(0, \tau_{\max }\right]=(0,0.2 \mathrm{~s}] \cup(0.2 \mathrm{~s}, 0.6 \mathrm{~s}] \cup(0.6 \mathrm{~s}, 0.7 \mathrm{~s}]$.

According to results of [7], we have $\bar{a}=10$ and $\delta(1)=0.2, \quad \delta(2)=0.6, \quad \delta(3)=-0.4$, $\delta(4)=2.1, \delta(5)=6.8, \quad \delta(6)=-3, \delta(7)=2$, $\delta(8)=1.7, \delta(9)=4.5, \delta(10)=2.3$, and the networked feedback controller for every

$$
\bar{\delta}=1.3 \text { is } F=\left[\begin{array}{ll}
-1.4 \times 10^{-2} & 2.3 \times 10^{-3}
\end{array}\right] \text {. Next, we }
$$

assume that the working status of NCSs are switched between in the normal case and un-normal case. The random generated network-induced delay and the number of successive packet dropout are taken larger than the parameter $\tau_{\text {max }}=0.7 \mathrm{~s}$ and $d_{\text {max }}=4$, which are $\tau_{\text {max }}=0.8 \mathrm{~s}$, $d_{\text {max }}=5$.

According to Theorem 1 in this paper, we can calculate the parameter index $\zeta_{\text {max }}=0.7$ and the correspondingly best combination of $L(j)$, which are described as

$$
L(9)=\left[\begin{array}{ll}
0.1 \times 10^{-4} & 3.1
\end{array}\right] \text { and } L(10)=\left[\begin{array}{ll}
0.25 & 2 \times 10^{-4}
\end{array}\right] .
$$

Furthermore, we consider the dynamic behavior of the NCSs which is witching between the normal case and the unnormal case. The initial condition response under the network-induced delay and packet dropouts is plotted in Fig. (2) and Fig. (3), where $x(0)=\left[\begin{array}{ll}50 & -30\end{array}\right]$ and $x(0)=\left[\begin{array}{ll}5 & -3\end{array}\right]$. It shows that under normal case and un-normal case, the proposed control strategy works well. Therefore, based on the above analysis, it is obvious that the proposed control strategy in this paper is effective, see Fig. (3).

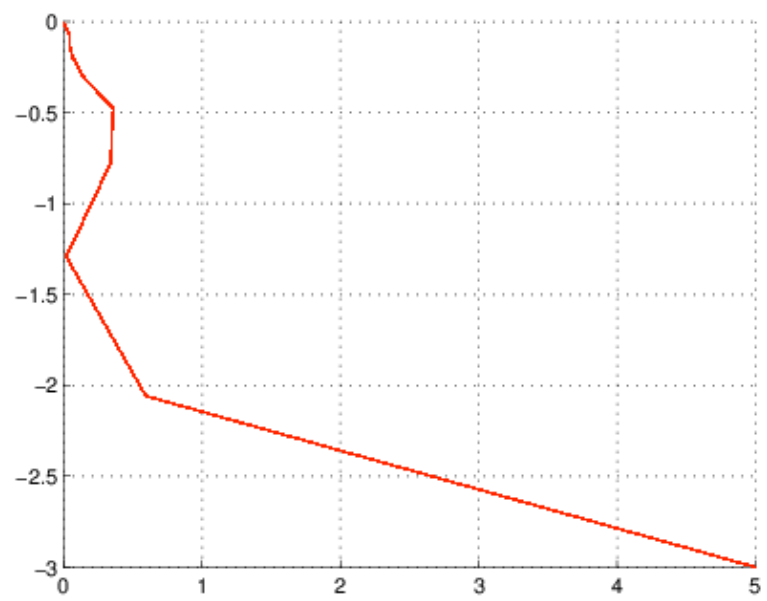

Fig. (3). State response curve of NCSs with initial conditions.

\section{CONCLUSION}

In this paper, we have considered the stability and controller design problem of networked control systems (NCSs) 
with two different working status. The obtained results can be considered as the development of the theory in [7], which only considers the normal case. Under the un-normal case, there are two goals which have been achieved for designing: one is to simulate the initial system for restarting the normal case; the other is to ensure the stability of NCSs as much as possible. By proposing a necessary and sufficient condition for guaranteeing the stability of the NCSs under the unnormal case, the dynamic behavior of the NCSs which is switching between the normal case and the un-normal case has been studied.

\section{CONFLICT OF INTEREST}

The authors confirm that this article content has no conflict of interest.

\section{ACKNOWLEDGEMENTS}

This work is supported by the China Postdoctoral Science Foundation Funded Project (2014M551110) and National Natural Science Foundation of China (61403073).

\section{REFERENCES}

[1] G. C. Walsh, H. Ye, and L. G. Bushnell, "Stability analysis of networked control systems," IEEE Transactions on Control System Technology, vol. 10, pp. 438-446, 2002.

[2] W. Zhang, M. S. Brabicky, and S. M. Philips, "Stability of networked control systems," IEEE Control System Magazine, vol. 21, pp. 84-99, 2001

[3] J. L. Xiong and J. Lam, "Stabilization of linear systems over network with bounded packet loss," Automatica, vol. 43, pp. 43-87, 2007.

[4] Y. B. Zhao, G. P. Liu, and D. Rees, "Modeling and stabilization of continuous-time packet-based networked control systems," IEEE Transactions on Systems, Man, and Cybernetics-Part B, vol. 39, pp. 1646-1652, 2009.
[5] H. G. Zhang, J. Yang, and C. Y. Su, "T-S fuzzy model based robust $\mathrm{H}$-infinite design for networked control systems with uncertainties," IEEE Transactions on Industrial Informatics, vol. 3, pp. 289301, 2007.

[6] M. G. Rivera, and A. Barreiro, "Analysis of networked control systems with drops and vriable delays," Automatica, vol. 43, pp. 2054-2059, 2007.

[7] H. G. Zhang, G. T. Hui, and Y. C. Wang, "Stabilization of networked control systems with piecewise constant generalized sampled-data hold function," International Journal of Innovative Computing, Information and Control, vol. 9, pp. 1159-1170, 2013.

[8] C. Peng, D. Yue, and Y. C. Tian, "Delay distribution based robust H-infinite control of networked control systems," Asian Journal of control, vol. 12, no. 1, pp. 1-12, 2010.

[9] G. T. Hui, H. G. Zhang, Z. N. Wu, and Y. C. Wang, "Control Synthesis Problem for Networked Linear Sampled-data Control Systems with Band-limited Channels," Information Sciences, vol. 275, pp. 385-399, 2014.

[10] R. H. Gielen, S. Olaru, M. Lazar, W. P. M. H. Heemels, N. V. D. Wouw, and S. I. Niculescu, "On polytopic inclusions as a modeling framework for systems with time-varying delays," Automatica, vol. 46, pp. 615-619, 2010.

[11] G. T. Hui, J. Yang, and B. N. Huang, "Comments on "Quantized Control Design for Impulsive Fuzzy Networked Systems," Ieee Transactions On Fuzzy Systems, vol. 21, pp. 987-988, 2013.

[12] G. T. Hui, H. G. Zhang, Y. C. Wang, and M. Dong, "Design of networked control systems with partly unknown markovian transition probabilities," In: IEEE World Congress on Computational Intelligence, 2010, pp. 1582-1587.

[13] H. G. Zhang, Z. S. Wang, and D. R. Liu, "A comprehensive review of stability analysis of continuous-time recurrent neural networks," IEEE Transactions on Neural Networks and Learning Systems, vol. 25, 1229-1262, 2014.

[14] H. G. Zhang, C. B. Qing, B. Jiang, and Y.H. Luo, "Online adaptive policy learning algorithm for H-infinite state feedback control of unknown affine nonlinear discrete-time systems," IEEE Transactions on Cybernetics, vol. 44, pp. 2706-2718, 2014.

[15] H. G. Zhang, F. S. Yang, X. D. Liu, and Q. L. Zhang, "Stability analysis for neural networks with time-varying delay based on quadratic convex combination, "IEEE Transactions on Neural Networks and Learning Systems, vol. 24, pp. 513-521. 2013.

(C) Hui et al.; Licensee Bentham Open.

This is an open access article licensed under the terms of the (https://creativecommons.org/licenses/by/4.0/legalcode), which permits unrestricted, noncommercial use, distribution and reproduction in any medium, provided the work is properly cited. 